\title{
Analytical Solution of Non-Isothermal Couette Flow between two Plates.
}

\author{
A.W. Ogunsola, B. A. Peter \\ Department of Pure and Applied Mathematics, LAUTECH,Ogbomoso, Nigeria.
}

\begin{abstract}
We present a paper on the non-isothermal couette flow between two plates. We investigate fluid flow between two fixed parallel horizontal plates. The fluid is assumed to depend on temperature. We model a viscous fluid, it is assumed that the viscosity of the fluid is linear and the thermal conductivity is a linear function of the temperature. We investigate the properties of the velocity and we show that the temperature and velocity fields have two solutions for some viscosity and the results were discussed.

Keywords: Non-Isothermal; Couette flow; Parallel Horizontal Plates and Non-Newtonian.
\end{abstract}

\section{Introduction:-}

Rheological measurements of viscosity and normal stresses in non-Newtonian fluids have attracted attention in the last few decades. These measurements are mainly performed on the basic assumptions that viscosity shear flow in geometrical annuli is steady, uniform axial and isothermal.

Abu et al [1] studied non-isothermal spherical couette flow of Oldroyd-B fluid. Zhizhin [2] treated the nonisothermal couette flow of a non-Newtonian fluid under the action of a pressure gradient. Wendi[3] studied the general solution of the couette flow profile. Lyubimov et al [4] considered the non-isothermal two phase flow in a closed cavity, where one of phases is gas(or liquid) and another phase consists of solid particles.Sabiki and Ayeni [5]studied a note on non-isothermal couette flow. Tan,W.C.et al [6] also investigated unsteady flows of a viscoelastic fluid with the fractional Maxwell model between two parallel plates.

In this paper ,we consider the case when the fluids viscosities depend much on the temperature and the effects of variable viscosity and thermal conductivity on the flow behavior.

\section{Mathematical Formulation}

The governing equations are momentum and energy equations as proposed by [5] .

$$
\begin{gathered}
v_{0} \frac{d u}{d y}=\frac{d}{d y}\left[\mu_{0}\left(\frac{T}{1+T}\right) \frac{d u}{d y}\right] \\
u(0)=0, u(h)=U \\
\rho_{C P} v_{0} \frac{d T}{d y}=\frac{d}{d y}\left[k_{0} T(1+T) \frac{d T}{d y}\right] \\
T(0)=T_{0}, T(h)=T_{1}
\end{gathered}
$$

where

$c p=$ specific heat,$U=$ velocity component in the $\mathrm{x}$-direction

$\mathrm{k}$ is the thermal conductivity, $\rho$ is the density, $\mu$ is the dynamic viscosity,

$h=$ distance between two parallel plates, $u=$ velocity,

$v_{0}=$ constant vertical velocity and $T=$ Temperature, $T_{0}=$ Temperature at the origin.

\section{Method Of Solution}

In order to solve the problem and keep it tractable, the set of non-linear ordinary differential equations $(2.1) \&(2.3)$ with boundary conditions in $(2.2) \&(2.4)$ have been solved analytically and numerically.

From Eq.(2.3) we integrate to get

$$
\rho c p v_{o} T+c=k_{0} T(1+T) \frac{d T}{d y}
$$

From Eq.(3.1) we collect like terms to get 
$\left.d y=k_{0} T(1+T) / \rho c p v_{0} T+c\right] d T$

From Eq.(3.2)

Let $\rho c p v_{0}=a, c=a, k_{0}=1$

$d y=\frac{T}{a} d T$

From Eq.(3.3) we integrate to get

$T^{2}=2 a y+c_{2}$

Where

$c_{2}=2 a c_{1}$

From Eq.(3.4) together with the boundary condition to get

$T=\left[2\left(\frac{T_{1}^{2}-T_{0}^{2}}{2 h}\right) y+T_{0}^{2}\right]^{1 / 2}$

From Eq.(3.6)

Let $T_{1}=1, h=1, T_{0}=0$

$T=y^{1 / 2}$

From Eq.(2.1) we integrate to get

$\left(\frac{1+T}{T}\right) d y=\frac{\mu_{0}}{v_{0} u+c}$

we substitute for T in Eq.(3.8) to get

$\left(1+y^{-1 / 2}\right) d y=\frac{\mu_{0}}{v_{0} u+c} d u$

from Eq.(3.9) we integrate to get

where $A=\frac{e^{\frac{k v_{0}}{\mu_{0}}}}{v_{0}}, B=\frac{c}{v_{0}}$

from Eq.(3.10) together with the boundary conditionswe get

$$
\begin{aligned}
& c\left(e^{\frac{2+\sqrt{1}\left(v_{0}\right)}{2\left(\mu_{0}\right)}}-1\right)=v_{0} U \\
& \text { Let } \\
& \mu_{0}=0.35, v_{0}=-1.0
\end{aligned}
$$

From Eqs.(3.12)-(3.13) together with the boundary conditions to get 
$u=-0.158\left[e^{-2.86\left(\frac{\sqrt{y}+2 y}{2}\right)}-1\right]$

\section{Results}

Analytical solutions of Eqs.(3.7) and (3.14) together with the boundary conditions (2.2) -(2.4)were provided for various parameters in the flow equations.

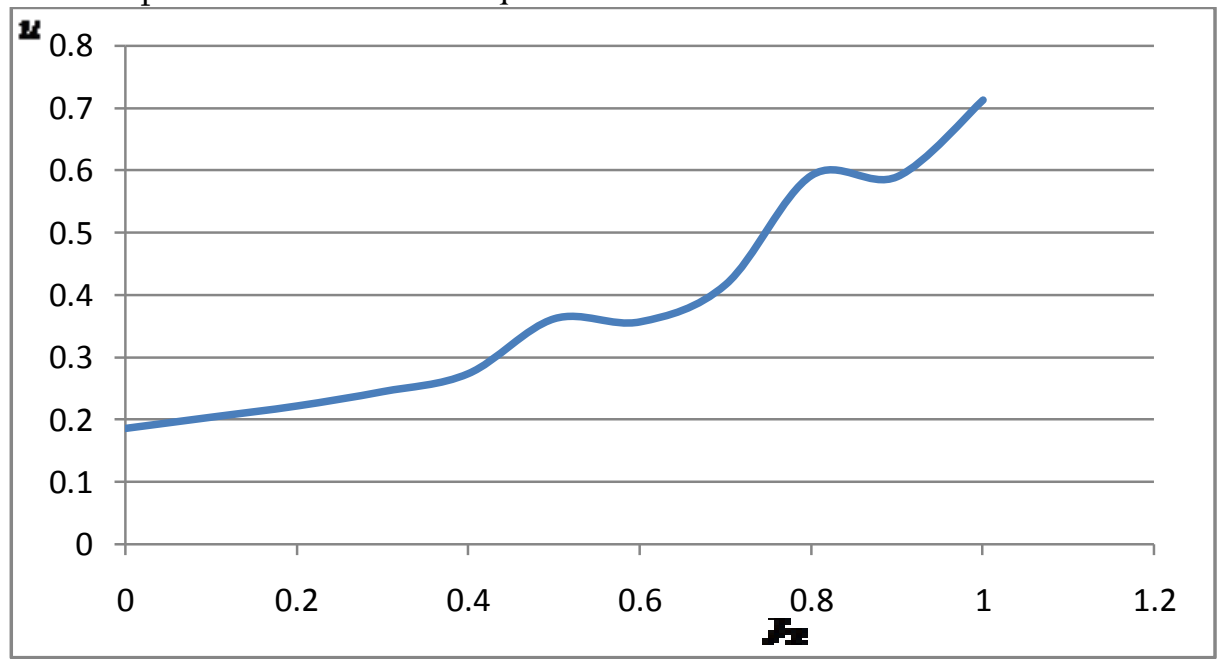

Fig4.1: Graph of the velocity function $u$ against the similarity variable $h$.

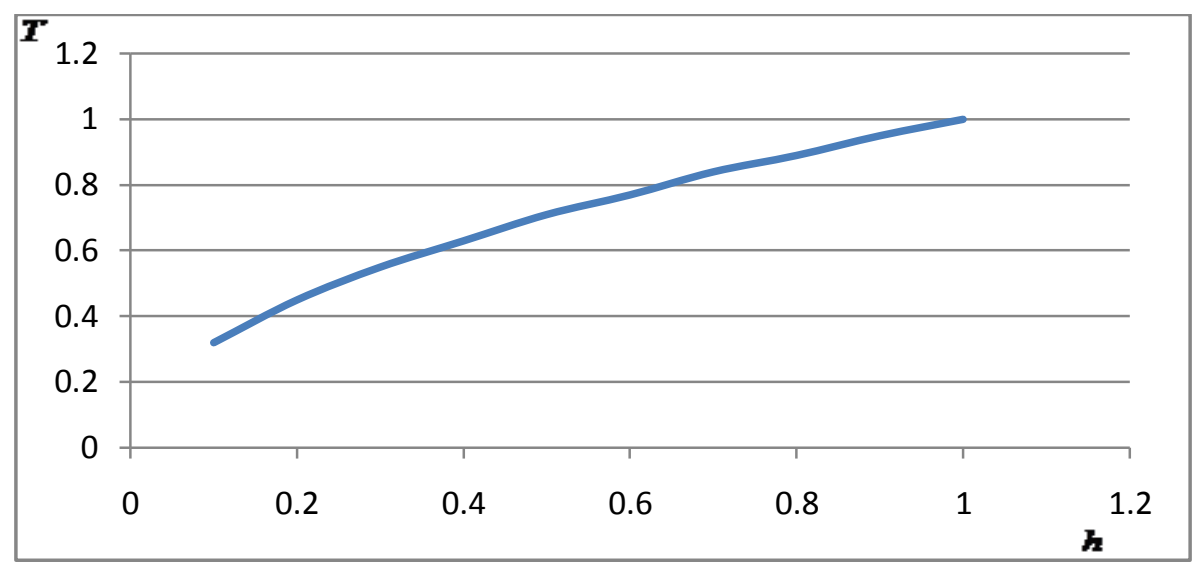

Fig4.2: Graph of the velocity function $T$ against the similarity variable $h$.

We now proceed to solve equations (2.1) and (2.3) subject to (2.2) and (2.4) numerically using

Galerkin-Weighted Residual Method as follows:

$$
\text { let } u=\sum_{i=0}^{2} A_{i} e^{y}, \theta=\sum_{i=0}^{2} B_{i} e^{(-i / 4) y}
$$

A maple14 pseudo code was used to perform the iterativecomputation and results are presented in Figures 2 and 3 as follows: 


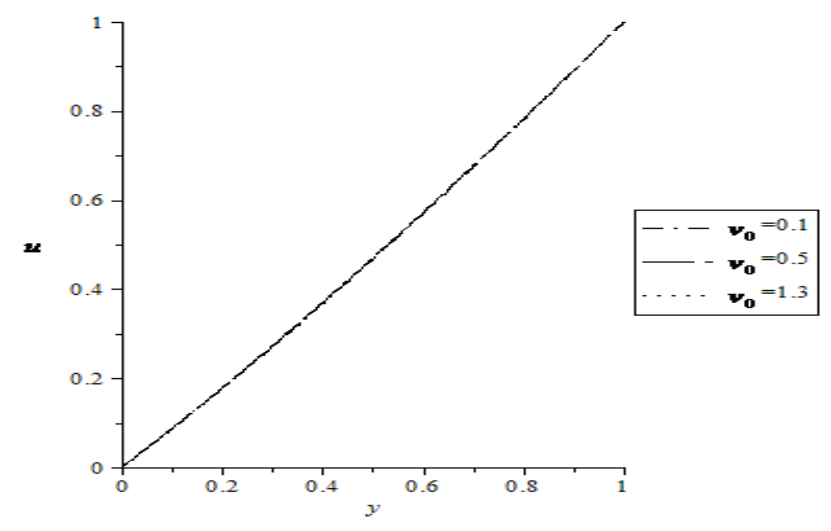

Figure 3: Graph of the velocity function $u$ against the similarity variable $y$ when

$$
c=0.5, \mu_{0}=0.5
$$

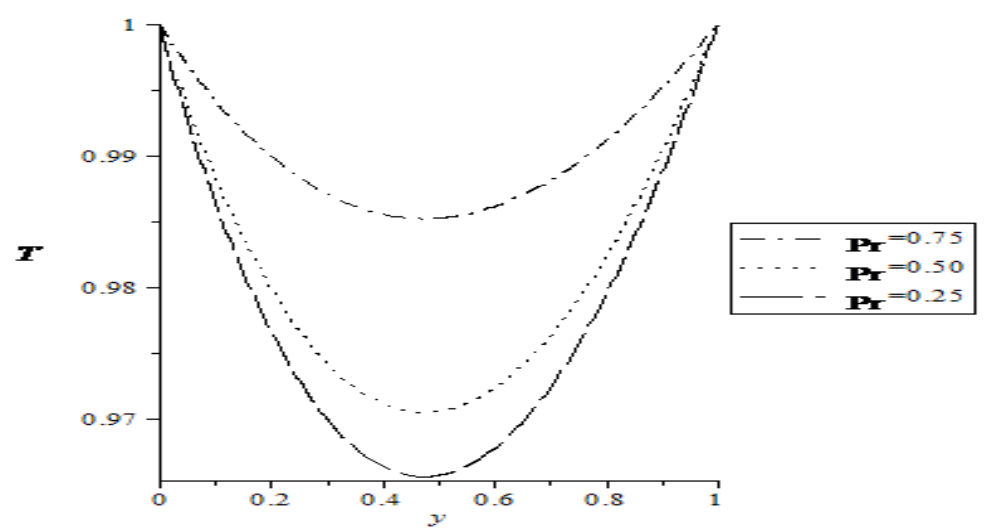

Figure 4: Graph of the temperature function $\theta$ against the similarity variable $y$ when

$c=0.5, \mu_{0}=0.5$

\section{Conclusion}

We obtained a suitable expression for non-isothermal steady- state flow of a Newtonian fluid between two parallel plates. It is assumed that the upper plate moves with a constant velocity $U$ and set the fluid particles moving in the direction parallel to plates while the lower plate remains stationary. The flow is considered steady, one-dimensional and compressible. To examine the flow behavior the velocity of moving plate is set at $U=1$, the temperatures at moving and stationary plateare $T_{1}=1, h=1, T_{0}=0$.

The velocity and temperature distributions along the $y$-direction of the parallel plates are obtained. It is seen from Figures $1 \& 3$ that variable thermal conductivity and viscosity increases the velocity of flow. It is seen from Figure 2 that variable thermal conductivity increases the temperature of the flow system. It is noticed from figure 4 that the temperature profile decreases as Pr number decreases.

It is also observed that minimum point exist in figure 4. 


\section{References}

[1] Abu-EL HassanA,Zidan M and Moussa M.M.(2008). Non-isothermal spherical couette flow ofOldroyd-B fluid,Z. AngewMath.Phys .59,1-22.

[2] Zhizhin G.V.(1981) Non-isothermal couette flow of a non-Newtonian fluid under the action of a pressure gradient .Journal of Applied Mechanics and Technical Physics. 22,306-309.

[3] Wendi M.C.(1999). General solution of the couette flow profile. Journal of Physical Review.60,6192-6194.

[4] D.V. Lyubimov,D.A.Bratsun,T.P. Lyubimova and B.Roux and V.S.Teplov, Non -isothermal flows of Dusty media,Third International Conference on Multiphase Flow, ICMF98,Lyon.France.(1998),1-5.

[5] I.A. Sabiki and R.O. Ayeni .Non-isothermal couette flow between two plates. Unpublished M.Sc.Thesis,OlabisiOnabanjo University,Ago-Nigeria.

[6] W.C.Tan,W.Pan and S.Asghar.(2004) .A note on the unsteady flows of a viscoelastic fluid with the fractional Maxwell model between two parallel plates. Int. J. Non-Linear Mech.44, 645-650.

[7] H.Xue, H.M.Jiand C.Shu.(2000).Analysis of micro-Couette flow using the Burnett equations. Int. J. Heat and Mass Transfer .44(2001), 4139-4146. 